\title{
The impact of ageing and sex on the association between sleepiness and sleep disordered breathing
}

\author{
Mary J. Morrell*, Laurel Finn\#", Alison McMillan* and Paul E. Peppard\#
}

\begin{abstract}
The aim of our study was to investigate age-related changes in sleepiness symptoms associated with sleep disordered breathing (SDB).
\end{abstract}

Wisconsin Sleep Cohort participants were assessed using polysomnography, the Epworth Sleepiness Scale (ESS) and the multiple sleep latency test (MSLT). SDB was defined as an apnoea/hypopnoea index $\geqslant 15$ events $\cdot h^{-1}$, and sleepiness as ESS $\geqslant 10$ and MSLT $\leqslant 5$ min. Odds ratios were calculated using generalised estimating equations associating sleepiness with SDB, and conditional logistic regression examining changes in longitudinal sleepiness status (ESS only). Models were a priori stratified by sex.

ESS was measured in 1,281 participants and MSLT in 998 at multiple time-points (ESS n=3,695; MSLT $n=1,846)$. Significant interactions were found between SDB and age in males, but not females. The odds ratios modelled for sleepiness in a 40 -yr-old male with SDB were significant compared to a male without SDB (ESS 2.1 and MSLT 2.9); however, these associations were not significant at 60 yrs of age. The within-subject odds ratio for sleepiness was also significant at 40 yrs of age (OR 3.4), but not at 60 yrs of age.

The age-related reductions in the association between sleepiness and SDB may have clinical implications for the diagnosis and treatment of SDB in older people as sleepiness is often used as a therapeutic target.

\section{KEYWORDS: Ageing, obstructive sleep apnoea, sleep disordered breathing, sleepiness}

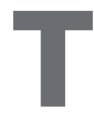
he prevalence of sleep disordered breathing (SDB) in older people is almost double that of younger people, with $\sim 20 \%$ of people aged $>65$ yrs having moderate-to-severe SDB when measured in a community sample; although a plateau occurs in the prevalence after the age of $60 \mathrm{yrs}$ [1]. Using a more liberal measure of SDB (apnoea/hypopnoea index (AHI) cut-off of $>5$ events $\cdot h^{-1}$ ) estimates range up to $56 \%$ in community populations and $70 \%$ in clinics and nursing homes (age 65-101 yrs) [2]. Despite this high prevalence, the age-related changes in the symptoms and consequences of SDB are poorly understood. For example, if sleepiness is the cardinal symptom of SDB in younger people, is this still the case in older people, where ageing per se may be associated with sleepiness?

In some studies, older people with SDB have been found to have minimal symptoms of subjective and objective sleepiness [3], while others have reported excessive sleepiness [4, 5]. However, in the former study by PHILLIPS et al. [3], the participants had a mean \pm SD age of $64 \pm 8.6$ yrs compared to $>70$ yrs in the latter studies $[4,5]$. In the Sleep Heart Health Study the strength of the association between SDB and sleepiness was similar in older ( $>65 \mathrm{yrs})$ and younger $(\leqslant 65 \mathrm{yrs})$ patients [6]. If symptoms of sleepiness are modified by age in people with SDB, this could have wider implications for the planning of healthcare services, since sleepiness is currently a therapeutic target for the treatment of SDB using continuous positive airway pressure (CPAP) [7]. The majority of studies investigating the effect of CPAP treatment on sleepiness have enrolled patients with SDB aged 44-58 yrs [7]. We aimed to investigate the strength of the association between symptoms of daytime sleepiness and SDB across the age spectrum using data from the Wisconsin Sleep Cohort.

In a recent study, also from the Wisconsin Sleep Cohort, SDB was predictive of cardiovascular mortality in participants with and without sleepiness [8]. The authors concluded that the high cardiovascular risk in untreated severe SDB suggested

\section{AFFILIATIONS}

*Academic Unit of Sleep and

Ventilation, National Heart and Lung Institute, Imperial College London, Royal Brompton Hospital, London, UK.

\#Dept of Population Health Sciences, University of Wisconsin-Madison, Madison, WI, USA.

\section{CORRESPONDENCE}

M.J. Morrell

Academic Unit of Sleep and Ventilation

Royal Brompton Hospital

Sydney Street

London

SW3 6NP

UK

E-mail: m.morrell@imperial.ac.uk

Received:

Oct 122011

Accepted after revision:

Dec 102011

First published online:

Jan 122012

European Respiratory Journal

Print ISSN 0903-1936

Online ISSN 1399-3003 
that SDB treatment should not be contingent on symptoms of daytime sleepiness. Other authors have also proposed that SDB without daytime symptoms should be treated to reduce cardiovascular risk [9]. This may be particularly relevant in older people, in whom the cardiovascular risk is greater. However, a counter point is that the presence of moderate SDB could provide a survival advantage in older patients [10]. Older patients with SDB may also benefit from improvements in cognitive function, independent of daytime sleepiness if treated for SDB; especially if they are at greater risk of cognitive decline that is independent of sleepiness [11]. Taken together these studies suggest that the appropriateness of using sleepiness to guide treatment choices in older populations with a higher absolute risk for cardiovascular events, cognitive impairment and mortality is questionable. We tested the hypothesis that ageing would be associated with a reduction in the symptom of sleepiness in patients with SDB.

\section{METHODS}

\section{Participants}

In 1988, employees of four state agencies in Wisconsin, USA, aged 30-60 yrs, were surveyed by post regarding their sleep habits. From these data, a sampling frame was constructed and 3,170 randomly selected respondents were invited to participate in the Wisconsin Sleep Cohort. Participants were studied overnight. Repeat studies occur at 4-yr intervals. In August 2011, there were 1,521 (48\%) participants with at least one adequate sleep study. The primary reason for non-participation was the burden of an overnight sleep study. One to six sleep studies per participant were available for analysis. Follow-up rates (calculated from average rates of refusal to participate in follow-up studies) were $\sim 80 \%$, although recruitment for followup studies continues to accrue. Consent documents for the ongoing Wisconsin Sleep Cohort Study were approved by the University of Wisconsin-Madison Health Sciences Institutional Review Board. All participants gave written, informed consent.

\section{Polysomnography}

Overnight polysomnography (Polygraph model 78; Grass Instruments, Quincy, MA, USA) plus other clinical assessments were carried out for each participant. Sleep state was determined by electroencephalography (EEG), electrooculography and electromyography. SDB events were assessed using arterial oxygen saturation (model 3740; Ohmeda, Englewood, CO, USA), oral and nasal airflow monitored by thermocouples (ProTec Inc., Hendersonville, TN, USA), nasal air pressure (Validyne Engineering Corp., Northridge, CA, USA), and thoracic and abdominal respiratory motion recorded by respiratory inductance plethysmography (Respitrace; Ambulatory Monitoring, Ardsley, NY, USA).

Sleep state and respiratory events were scored by trained sleep technicians using the conventional Rechtschaffen and Kales criteria. An apnoea was defined as the cessation of airflow lasting $\geqslant 10 \mathrm{~s}$, and a hypopnoea was defined as a discernible reduction in the sum of thoracic plus abdominal respiratory inductance plethysmography amplitude associated with a $\geqslant 4 \%$ reduction in oxygen saturation. The average number of apnoea plus hypopnoea events per hour of sleep defined the AHI.

\section{Assessment of sleepiness}

The sleepiness assessments used in this study were the subjective Epworth Sleepiness Scale (ESS) questionnaire [12] and the objective multiple sleep latency test (MSLT) [13]. These were performed on the same day as the polysomnography. These tests were added to the Wisconsin Sleep Cohort study protocol at different times. The ESS was first used in October 1993 and data were available in 1,281 participants (693 males, 588 females), with 3,695 total sleep studies $(270,211,310,373$ and 117 participants with one, two, three, four and five studies, respectively). Subjective sleepiness was defined as an ESS $\geqslant 10$, alternative cut-off points were examined to determine if our results were sensitive to the choice of cut-off point.

The MSLT research protocol was performed between June 1989 and August 2003. Data were available in 998 participants (551 males, 447 females), with 1,846 total sleep studies (404, 376, 182 and 36 participants with one, two, three and four studies). Participants were allowed four nap opportunities during the day at 2-h internals in a darkened room. The nap opportunity was stopped if the EEG showed the participant had fallen asleep or after $20 \mathrm{~min}$ if sleep did not occur. Objective sleepiness was defined as an MSLT $\leqslant 5 \mathrm{~min}$.

\section{Covariate measurements}

All participants were interviewed at each visit regarding their usual sleep time (average hours of sleep per day including naps), medical history (including self-reported of physician diagnosed comorbidities, congestive heart failure, myocardial infarction, revascularisation procedures, arthritis and stroke), chronic joint and/or back pain, medication use (coded into pharmacologic categories), alcohol use (drinks per week), smoking habits (current and past) and caffeine use (daily consumption). Age and body mass index (BMI; $\mathrm{kg} \cdot \mathrm{m}^{-2}$ ) were also measured. The Zung self-reported depression scale was completed, which is a 20-item survey assessing depressive symptoms on a scale ranging from 25 to 100 ; scores $\geqslant 50$ were taken as mild or worse depressive symptomatology [14]. The StateTrait Anxiety Inventory scale was also completed [15]. This is a 40-item survey, with 20 items assessing state anxiety and 20 items assessing trait anxiety. Each scale ranges from 20 to 80 . Finally, hours of physical exercise was collected on the initial survey.

\section{Statistical analyses}

Analyses were performed with SAS software version 9.2 (SAS Institute, Inc., Cary, NC, USA). Due to the fact that our data uses multiple measurements per person, we examined loss to follow-up. Characteristics of sleepiness, SDB, age, BMI and sex were compared between participants with and without followup data $(n=1,011$ versus $n=270)$ using unpaired $t$-tests and Chisquared tests.

Two types of regression models were used to estimate odds ratios associating the presence of sleepiness with the presence of SDB; ESS $\geqslant 10$ or MSLT $\leqslant 5$ min were modelled separately. Generalised estimating equation models were fitted to all data, including multiple sleep studies per participant, if available. These models are analogous to logistic regression models, with techniques for repeated measures. The resulting odds ratios represent the weighted averages of the cross-sectional and longitudinal associations, between sleepiness and SDB, while 
accounting for within-subject correlation due to the use of multiple sleep studies per participant [16].

Conditional (intrasubject) logistic regression was used to estimate the odds of changing sleepiness status between visits (i.e. having an ESS $<10$ at a study visit, and an ESS $\geqslant 10$ at a subsequent visit, or vice versa) associated with also changing SDB status between visits [16]. This purely longitudinal approach was used only with ESS as there were insufficient participants with multiple MSLTs. The within-subject approach accommodated the participants that were initially identified as sleepy, but were no longer sleepy at a later visit. The conditional model implicitly controls for fixed within-person characteristics, such as sex and genetic profile [16]. All models were a priori stratified by sex. Within sex-stratified models the interaction terms for SDB multiplied by age were fitted to test the primary hypothesis that the association between sleepiness and SDB reduces with age. A significantly negative interaction coefficient would provide evidence consistent with a diminishing association between sleepiness and SDB with increasing age.

SDB was categorised as either absent $(\mathrm{AHI}<15)$ or present (AHI $\geqslant 15$ or use of continuous positive airway pressure (CPAP) therapy). The primary outcome variables were the presence or absence of excessive daytime sleepiness defined as: ESS $\geqslant 10$ or MSLT $\leqslant 5 \mathrm{~min}$. Supplemental analyses were performed to examine alternative outcome characterisations for sleepiness (ESS cut-off point of nine or 11; ESS and MSLT modelled as a continuous outcomes) and SDB (AHI modelled as a continuous variable; exclusion of subjects using CPAP from analyses). We found no meaningful differences in the alternative models compared to the results presented here. Covariates (BMI, comorbidities, alcohol and cigarette use, caffeinated beverage consumption, physical exercise, depressive or anxious symptomology, hypnotic medication use, chronic joint and/or back pain and self-reported usual sleep time) which substantially altered regression coefficients for SDB were retained in final models. We were not able to examine the effect of confounding for chronic joint/back pain or arthritis for the MSLT models, as the data collection of this information was added into the study later and the sample size was not sufficient to address this question.

\section{RESULTS}

The characteristics of the participants in whom we examined the symptom of sleepiness using the ESS and the MSLT are described in tables 1 and 2, respectively. There were no substantial differences between the samples in which ESS and MSLT were measured. In both samples, participants with SDB were older and had higher BMI, compared to those without SDB. The participants with SDB also had a higher prevalence of depressive symptomology and cardiovascular diseases. Additionally, we examined differences in participants with and without follow-up data and found no differences in ESS, $\mathrm{SDB}$, sex or BMI between the two groups. There was a statistically significant, but minimal difference in age between the two groups (50.4 yrs for participants with follw-up date versus 51.5 yrs for participants without follow-up data).

Both subjective and objective sleepiness symptoms were more prevalent in participants with SDB compared to those without. The partial correlation (rpartial) between age and ESS, adjusting for sex, BMI and AHI was -0.04 $(p=0.04)$. A stronger rpartial was found with age and MSLT, $0.10(\mathrm{p}<0.001)$.

In mixed longitudinal cross-sectional models, a significant interaction was found between SDB and age, predicting sleepiness in males but not in females. In males, the association between both subjective and objective sleepiness and SDB diminished significantly with age, adjusting for comorbidities, depressive symptomology and BMI. Further adjustments for other covariates had no effect and were not included in the final models. For subjective sleepiness, the male odds ratio decreased by $27 \%$ for each $10-y$ r increase in age. For objective sleepiness, the odds ratio decreased by $44 \%$ for each $10-y r$ increase in age.

The modelled associations between sleepiness and SDB calculated for participants aged 40 and 60 yrs are presented in table 3. Our data show that a 40-yr-old male with SDB has a 2.1 times greater odds of being subjectively sleepy, than a male without SDB. However, for a 60 -yr-old male the association between subjective sleepiness and SDB is diminished, and no longer statistically significant. Similarly, a 40-yr-old male with SDB has a 2.9 times greater odds of being objectively sleepy, than a male without SDB; however, for a 60-yr-old male the association is reduced and not significant. Females with SDB aged both 40 and 60 yrs had slightly elevated odd ratios of subjective and objective sleepiness, relative to females without SDB, but these were not statistically significantly.

In the purely longitudinal models that examined the relationship of sleepiness (among participants observed to change their sleepiness status between visits) and SDB status, 231 males with 281 studies were used because they changed sleepiness status during follow-up studies, and 188 females with 246 studies were used for the same reason. Similar results to the mixed longitudinal cross-sectional models were found. Specifically, a significant interaction was found between SDB and age predicting subjective sleepiness in males, but not in females. Estimates of the association between subjective sleepiness and SDB for males and females aged 40 and 60 yrs are presented in table 4 . As with the models presented in table 3, adjustment for comorbidities, depressive symptomology and BMI had no effect on the associations. A 40-yr-old male had a 3.4 times greater odds of having excessive subjective sleepiness at the study visits when they had SDB, compared to the visits at which they did not have SDB. There was no significant association between SDB and changing ESS sleepiness status for a 60-yr-old male or females of any age. However, females with SDB had 1.4 to 1.5 times greater subjective sleepiness in the purely longitudinal models. Although these values are not statistically significant, this may be an important elevation in the odds of sleepiness measured using ESS. Our study was underpowered to identify an elevated risk of this magnitude with statistical significance. Also of note among persons classified as having SDB was that males tended to have more severe SDB (median AHI among participants with $\mathrm{AHI} \geqslant 15$ events $\cdot \mathrm{h}^{-1}$ : males 24 versus females 21). This may, in part, explain why stronger associations between SDB and sleepiness were detected in males compared to females.

Participants who reported using CPAP therapy were categorised as having SDB and grouped with participants with AHI $>15$, regardless of measured AHI (tables 3 and 4). This was 
TABLE 1 Characteristics of subjects examined for subjective sleepiness using the Epworth Sleepiness Scale

\begin{tabular}{|c|c|c|c|c|c|}
\hline \multirow[t]{2}{*}{ Characteristic } & \multicolumn{2}{|c|}{ Females $^{\#}$} & \multicolumn{2}{|c|}{ Males" } & \multirow[t]{2}{*}{ Total } \\
\hline & $\mathrm{AHI}<15$ & $\mathrm{AHI} \geqslant 15$ or CPAP & $\mathrm{AHI}<15$ & $\mathrm{AHI} \geqslant 15$ or CPAP & \\
\hline Sleep studies $n$ & 1472 & 211 & 1617 & 395 & 3695 \\
\hline \multicolumn{6}{|l|}{ Age yrs } \\
\hline Minimum & 32 & 35 & 33 & 34 & 32 \\
\hline 3rd quartile & 61 & 62 & 62 & 65 & 62 \\
\hline Maximum & 81 & 77 & 80 & 78 & 81 \\
\hline Body mass index $\mathrm{kg} \cdot \mathrm{m}^{-2}$ & $31 \pm 7$ & $41 \pm 10$ & $29 \pm 5$ & $35 \pm 7$ & $31 \pm 7$ \\
\hline Epworth Sleepiness Score & $8 \pm 4$ & $9 \pm 4$ & $9 \pm 4$ & $10 \pm 4$ & $9 \pm 4$ \\
\hline Epworth Sleepiness Score $\geqslant 10$ & $525(36)$ & $93(44)$ & $683(42)$ & $211(53)$ & $1512(41)$ \\
\hline MSLT min $^{+}$ & $11 \pm 5$ & $10 \pm 6$ & $9 \pm 5$ & $9 \pm 5$ & $10 \pm 5$ \\
\hline Depression $^{5}$ & $450(31)$ & $102(48)$ & $273(17)$ & $85(22)$ & $910(25)$ \\
\hline Trait Anxiety Scale & $34 \pm 8$ & $35 \pm 9$ & $32 \pm 8$ & $32 \pm 8$ & $33 \pm 9$ \\
\hline Comorbid conditions & $74(5)$ & $15(8)$ & $162(10)$ & $84(21)$ & $335(9)$ \\
\hline Stroke & $25(2)$ & $5(2)$ & $14(1)$ & $12(3)$ & $56(2)$ \\
\hline Heart failure & $10(1)$ & $2(1)$ & $8(0.5)$ & $8(2)$ & $28(1)$ \\
\hline Cardiovascular disease ${ }^{f}$ & $44(3)$ & $10(5)$ & $150(9)$ & $74(19)$ & $278(8)$ \\
\hline Arthritis $^{\# \#}$ & $380(35)$ & $104(57)$ & $230(20)$ & $101(32)$ & $815(30)$ \\
\hline Chronic joint/back pain $\# \#$ & $253(23)$ & $61(33)$ & $166(14)$ & $61(19)$ & $541(20)$ \\
\hline Current smoker & $175(12)$ & $21(10)$ & $190(12)$ & $42(11)$ & $428(12)$ \\
\hline Physical activity per week $h$ & $2 \pm 3$ & $2 \pm 2$ & $3 \pm 3$ & $2 \pm 2$ & $2 \pm 3$ \\
\hline Hypnotic medication use & $125(8)$ & $18(8)$ & $62(4)$ & $24(6)$ & $229(6)$ \\
\hline Alcoholic drinks per week & $1(0-36)$ & $0(0-17)$ & $3(0-42)$ & $2(0-32)$ & $2(0-42)$ \\
\hline
\end{tabular}

Data are presented as mean $\pm \mathrm{SD}, \mathrm{n}(\%)$ or median (range), unless otherwise stated. Data are reported on the number of sleep studies, with most subjects contributing to multiple studies. AHI: apnoea/hypopnoea index; CPAP: continuous positive airway pressure; MSLT: multiple sleep latency test; NA: not available. ${ }^{\#:} \mathrm{n}=1,683$;

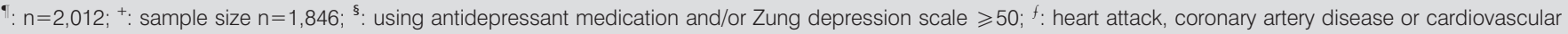
surgical procedure; $\#$ : these questions were added after the study began and are available in a subset.

carried out because it was not possible to estimate SDB in participants who were using CPAP during the study, and it was assumed participants were more likely to have been prescribed CPAP treatment if they were sleepy (for a given level of SDB severity). To assess the impact of CPAP use on our findings, the analyses were repeated excluding the participants that were using CPAP. In the model that included only males not using CPAP, the coefficients changed by $<10 \%$ compared to those estimated in presented models. Furthermore, in the model with CPAP users excluded, the results were the same; there was a relationship between sleepiness and SDB at age $40 \mathrm{yrs}$ and the association reduced as age increased. For the females the results remained non-significant in the subset with CPAP users removed.

\section{DISCUSSION}

The main finding of this large study, incorporating $>3,000$ measurements of subjective sleepiness and nearly 2,000 objective sleepiness measurements, was that the association between sleepiness and SDB reduces with age. A two-fold increase in subjective sleepiness was present in males with moderate-tosevere SDB at 40 yrs of age, but not at 60 yrs of age. In females, SDB was not associated with statistically significant increased sleepiness at any age, although a clinically significant association cannot be ruled out. Objective sleepiness measurements showed an even greater three-fold increase in the odds of sleepiness in younger males with SDB, compared to those without SDB; again in older people no relationship was found between SDB and objective measures of sleepiness.

The fact that both subjective and objective measures of daytime sleepiness reduced with age in patients with moderate-to-severe SDB strengthens our findings, and supports the suggestion that ageing per se is associated with a reduction in sleepiness symptoms in SDB. Current clinical practice recommends CPAP as an effective treatment for SDB, reducing symptoms of sleepiness in middle-aged patients with moderate-to-severe disease [7]. The results of our study imply that sleepiness may not be the most important target for SDB therapy in an older 
TABLE 2 Characteristics of subjects examined for objective sleepiness using the multiple sleep latency test (MSLT)

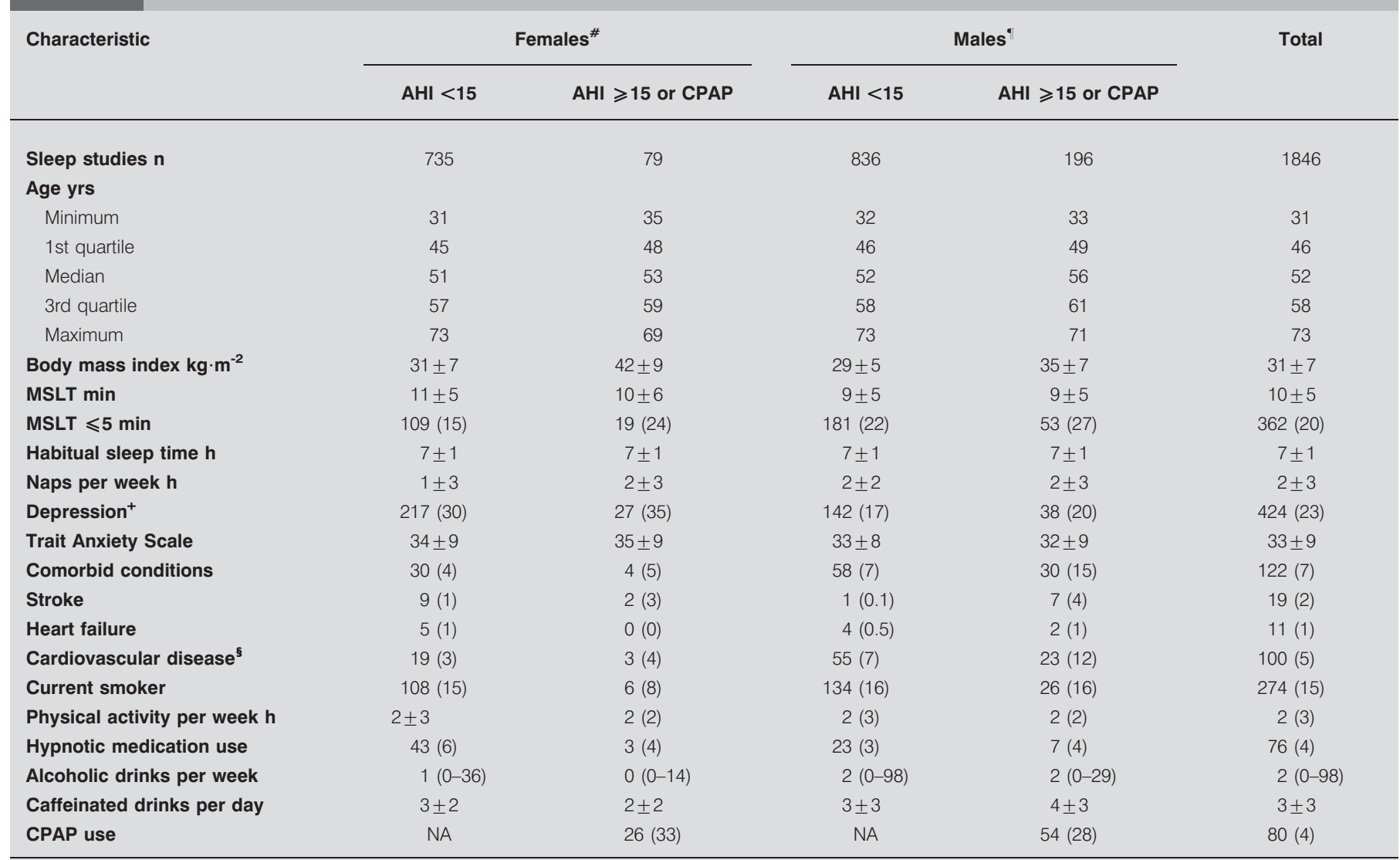

Data are presented as mean \pm SD, $n(\%)$ or median (range), unless otherwise stated. Data are reported on the number of sleep studies, with most subjects contributing to multiple studies. AHI: apnoea/hypopnoea index; CPAP: continuous positive airway pressure; NA: not available. ${ }^{\#}: n=814 ;{ }^{\circ}: n=1,032 ;{ }^{+}$: using antidepressant medication or Zung depression scale $\geqslant 50 ;{ }^{\S}$ : heart attack, coronary artery disease or cardiovascular surgical procedure.

population, and other factors such as quality of life or cognitive impairment could be more appropriate targets for therapeutic intervention.

\section{Sleepiness as a symptom of SDB}

The age-related reduction in sleepiness that we found in participants with SDB is consistent with previous studies that have shown reduced sleepiness in older people with SDB [3, 17]. This leads us once again to explore the notion of treating SDB based on symptoms of sleepiness. In younger people with SDB and little or no sleepiness mortality remains high [8], supporting the argument that SDB patients who are not sleepy should be offered treatment to reduce possible cardiovascular mortality. In older people the evidence for this treatment strategy is less clear [7]. Recent data shows that severe obstructive sleep apnoea (OSA) in older people increases all-cause mortality, specifically stroke, and that CPAP treatment reduced the increased cardiovascular mortality [18]. In other studies the relationship between SDB and cardiovascular mortality was only present in older OSA patients who were sleepy [19], and in some cases the association was reduced [20,21], possibly due to the activation of cardioprotective adaptive pathways, stimulated by chronic intermittent hypoxia [10]. Taken literally, these data appear to argue against treatment of non-symptomatic SDB in older people. Resolving this issue will require further studies in older people, adequately powered to establish the effect of SDB on both sleepiness and cardiovascular outcomes. There are currently no such published studies, and because excessive sleepiness (regardless of its cause) is associated with increased allcause mortality in older people [22] a randomised controlled trial is required.

SDB in older people may lead to cognitive decline, independent of sleepiness [11]; as with cerebrovascular sequelae this could be mediated by intermittent hypoxia [23]. The study by AYALON et al. [24] has recently shown that the combined effects of SDB and ageing produced significant impairments in cognitive function and neural activation, greater than either variable acting independently. These data are also in accordance with the findings obtained from an ageing animal model of OSA, where exposure to chronic intermittent hypoxia produced impaired special learning and increased neural apoptosis in older rats [25]. If older brains are more susceptible to intermittent hypoxia, then SDB in older people could be associated with cerebral atrophy, leading to accelerated cognitive decline, and treatment strategies would need to be developed accordingly. In this respect a single cognitive test, or standardised 


\begin{tabular}{|c|c|c|c|c|}
\hline \multirow{3}{*}{ Sleepiness measure } & \multirow{3}{*}{$\begin{array}{l}\text { hing (SDB) pre } \\
\text { onal models } \\
\text { Sleep studies n }\end{array}$} & \multirow{3}{*}{$\begin{array}{l}\text { abjective } \\
\text { Age yrs }\end{array}$} & \multicolumn{2}{|c|}{ s by sex and age from mixed } \\
\hline & & & \multicolumn{2}{|c|}{$\mathrm{SDB}^{\#}$ versus no SDB } \\
\hline & & & Males & Females \\
\hline \multicolumn{5}{|l|}{ Subjective sleepiness } \\
\hline Females & 1683 & 60 & $1.1(0.9-1.4)^{\bullet}$ & $1.2(0.9-1.7)^{+}$ \\
\hline \multicolumn{5}{|l|}{ Objective sleepiness } \\
\hline \multicolumn{5}{|l|}{$\mathrm{MSLT} \leqslant 5 \mathrm{~min}$} \\
\hline Males & 1032 & 40 & $2.9(1.3-6.4)^{\S}$ & $1.2(0.4-3.7)^{f}$ \\
\hline Females & 814 & 60 & $0.7(0.4-1.3)^{\S}$ & $2.1(0.9-4.6)^{f}$ \\
\hline
\end{tabular}

battery, to assess functional impairment in older OSA patients would be of value [26].

\section{Symptoms of SDB in females}

In our study females with SDB did not have statistically significantly elevated odds of sleepiness, and the relationship between sleepiness and SDB was not influenced by ageing. In previous studies, females with SDB have reported more symptoms of sleepiness than males [27, 28]. This may, in part,

\begin{tabular}{|c|c|c|c|}
\hline TABLE 4 & $\begin{array}{l}\text { Sleep disorder } \\
\text { subjective slee } \\
\text { longitudinal ma } \\
\text { observed to ch } \\
\text { visits }\end{array}$ & $\begin{array}{l}\text { eathing (SD } \\
\text { s by sex ar } \\
\text { using subje } \\
\text { sleepiness }\end{array}$ & $\begin{array}{l}\text { edicting } \\
\text { e from } \\
\text { vho were } \\
\text { us between }\end{array}$ \\
\hline \multirow{2}{*}{$\begin{array}{l}\text { Subjective } \\
\text { sleepiness }\end{array}$} & \multirow[t]{2}{*}{ Age yrs } & \multicolumn{2}{|c|}{$\mathrm{SDB}^{\#}$ versus no SDB } \\
\hline & & Males $\pi$ & Females $^{+}$ \\
\hline \multirow[t]{2}{*}{ ESS $\geqslant 10$} & 40 & $3.4(1.2-9.7)^{\S}$ & $1.4(0.3-5.7)^{\prime}$ \\
\hline & 60 & $1.1(0.6-1.8)^{5}$ & $1.5(0.8-3.0)^{\prime}$ \\
\hline
\end{tabular}

Data are presented as odds ratio (95\% confidence interval), unless otherwise stated. ESS: Epworth Sleepiness Scale. ${ }^{*}$ : defined as an apnoea/hypopnoea index $\geqslant 15$ events $\cdot h^{-1}$ or self-reported continuous positive airway pressure use and adjusted for comorbidities, depressive symptoms and body mass index $\because: n=266 .{ }^{+}: n=211 .{ }^{\S}$ : in males, the odds ratio (OR) for SDB predicting ESS $\geqslant 10$ as a function of age can be expressed as: $\mathrm{OR}=e(\alpha+\beta \times($ age in yrs-30)), where $\alpha$ (the estimated SDB-ESS $\geqslant 10$ OR at age $30 \mathrm{yrs}$ ) is $1.8(95 \% \mathrm{Cl} 0.34-$ 3.3) and $\beta$ (the coefficient for the SDB-age interaction term in males) is estimated to be $-0.058(95 \% \mathrm{Cl}-0.11--0.011) .{ }^{f}$ : in females, the OR for SDB predicting ESS $\geqslant 10$ is e $(\alpha+\beta \times$ (age in yrs-30)), where $\alpha=0.26(95 \% \mathrm{Cl}-1.8$ 2.4) and $\beta=0.005$ (95\% Cl-0.069-0.078). reflect differences in the perception of sleepiness between males and females; with females often reporting more fatigue or tiredness [29]. It is notable that in a recent large crosssectional study of older people, females with OSA perceived themselves to be less sleepy than males with OSA, but more anxious and depressed [30].

\section{Mechanism for the age-related reduction in sleepiness in older people with SDB}

We speculate that age-related changes in the cause of SDB may be responsible for the reduced symptoms of sleepiness in older people with SDB. For example, ageing is associated with a lengthening of the upper airway [31] and descent of the hyoid bone [32]. The response of the genioglossus muscle to negative pressure is also reduced in older people compared to younger people [33], and less negative pressure is required to collapse the upper airway, independent of BMI in older people [34]. These changes in the structure and function of the upper airway are likely to increase collapsibility, and the subsequent number of apnoeas or hypopnoeas in older people. Furthermore, if the amount of negative intra-luminal pressure required to collapse the upper airway is less, a smaller perturbation in sleep state may be required to re-open the airway. This could lead to SDB with minimal sleep disturbance in older people; although conversely, frequency of arousals from sleep is increased in older people [35]. It may also be that less CPAP pressure is required to keep the upper airway open [36, 37].

SDB is prevalent in many age-related diseases such as heart failure [38] and stroke [39]. When SDB occurs with these comorbidities the symptoms of sleepiness are reduced despite profound sleep disruption [40]. The suppressed symptoms of sleepiness may be due to factors associated with the disease processes such as increased sympathetic nervous system activity in chronic heart failure (CHF) patients. However, in 
the present study we corrected for self-reported cardiovascular co-morbidity.

The perception of sleepiness may be confounded by expectations, or habituation to the increased sleep fragmentation that occurs with ageing. However, our age-related reduction in objective sleepiness with SDB does not support this suggestion. Daytime napping could be a factor in reducing the symptom of sleepiness. Older people are less likely to be employed full-time, thus, may have more nap opportunities, whereas younger people in full-time employment may have less nap opportunity for a given level of nocturnal sleep disruption (due to SDB or other causes). We have previously shown a reduction in daytime activity associated with a reduction in sleepiness in CHF patients with SDB [41]. Such life style modifications are likely to reduce the impact of nocturnal sleep disturbances on the symptom of sleepiness in SDB.

\section{Critique of methods}

To interpret the results of this study it is necessary to consider several factors. The natural history of the disease is unknown in our cohort, although the purely longitudinal models do give some insight to this issue. Nevertheless, the temporal relationship between the development of the sleepiness and the duration of the SDB is unknown. Participants in the Wisconsin Sleep Cohort are invited to return every 4 yrs. At 4 and 8 yrs the response rate was $74 \%$ and $84 \%$, respectively. In later years the dropout rate may be greater in people who develop severe sleepiness or co-morbidities, potentially making the sample less representative.

At any age, CPAP treatment of severe, symptomatic SDB may mask associations between sleepiness and SDB. In our analysis we categorised participants with CPAP as having SDB, regardless of measured AHI (if available), we also had to take measured sleepiness in these people at face value, i.e. we were unable to adjust for what their sleepiness would have been if their SDB was untreated. Although, our re-analyses excluding the CPAP users did not alter our findings, this does not preclude the possibility that we have underestimated the association between sleepiness and SDB by excluding people with both severe sleepiness and severe SDB (in the untreated state). However, this bias would probably operate in both younger and older participants, so should not affect our comparison of associations in older versus younger persons.

In the present study we used both subjective and objective measures of sleepiness. The relationship between ESS and MSLT measures of sleepiness has long been debated [42], especially in relation to treatment of SDB [43]. A recent systematic review of CPAP for the treatment of OSA syndrome concluded that improvements of subjective sleepiness (measured by ESS) could be found in moderate-to-severe patients, whereas no statistical difference could be found in the length of time participants took to fall asleep post-treatment (measured by the MSLT) [7]. In older people, there are currently no treatment studies reporting the changes in ESS with CPAP treatment. Although others have also found a reduction in both ESS and MSLT measures of sleepiness in older patients with SDB [36]. Therefore, although longitudinal changes in ESS need to be interpreted with caution we would argue that the agreement between objective and subjective measures of sleepiness strengthen the findings of our study.

\section{Conclusion}

Our analysis of this large data set from the Wisconsin Sleep Cohort study has shown an age-related reduction in the association between SDB and symptom of sleepiness in males, but not females. These findings may explain, in part, why the reported increased prevalence of SDB in older people is not reflected in clinical populations. The lack of sleepiness in older people with SDB also implies that other consequences of SDB, such as reduced cognitive function, may be relatively more important for health in this group. Our findings lead us to speculate that in future, composite scores may be needed to identify the risks and determine treatment responses to SDB in older people.

\section{SUPPORT STATEMENT}

This study was supported by the US National Institute of Health (grants R01HL62252, R01AG14124, RR03186 and 1UL1RR025011). A. McMillan was funded by a National Institute of Health research grant. The study was supported by the NIHR Respiratory Disease Biomedical Research Unit at the Royal Brompton and Harefield NHS Foundation Trust and Imperial College London (London, UK).

\section{STATEMENT OF INTEREST}

Statements of interest for M.J. Morrell and L. Finn can be found at www.erj.ersjournals.com/site/misc/statements.xhtml

\section{ACKNOWLEDGEMENTS}

The authors would like to thank T. Young (Dept of Population Health Sciences, University of Wisconsin-Madison, Madison, WI, USA) for her long time leadership of the Wisconsin Sleep Cohort Study and the expertise of K. Mae Hla (Dept of Medicine, University of WisconsinMadison), as well as K. Pluff, A. Rasmuson, N. Salzieder, K. Stanback, R. Stubbs and M. Sundstrom (all from Dept of Population Health Sciences, University of Wisconsin-Madison) and K. Cacic (Institute for Clinical and Translational Research, University of WisconsinMadison), as well as members of the PREDICT trial team (NIHR funded project, UK).

\section{REFERENCES}

1 Young T, Shahar E, Nieto FJ, et al. Predictors of sleep-disordered breathing in community-dwelling adults: the Sleep Heart Health Study. Arch Intern Med 2002; 162: 893-900.

2 Glasser M, Bailey N, McMillan A, et al. Sleep apnoea in older people. Breathe 2011; 7: 249-256.

3 Phillips BA, Berry DT, Schmitt FA, et al. Sleep-disordered breathing in the healthy elderly. Clinically significant? Chest 1992; 101: 345-349.

4 Ancoli-Israel S, Kripke DF, Klauber MR, et al. Sleep-disordered breathing in community-dwelling elderly. Sleep 1991; 14: 486-495.

5 Knight H, Millman RP, Gur RC, et al. Clinical significance of sleep apnea in the elderly. Am Rev Respir Dis 1987; 136: 845-850.

6 Gottlieb DJ, Whitney CW, Bonekat WH, et al. Relation of sleepiness to respiratory disturbance index: the Sleep Heart Health Study. Am J Respir Crit Care Med 1999; 159: 502-507.

7 McDaid C, Griffin S, Weatherly H, et al. Continuous positive airway pressure devices for the treatment of obstructive sleep apnoea-hypopnoea syndrome: a systematic review and economic analysis. Health Technol Assess 2009; 13: iii-iv, xi-xiv, 1-119: 143-274. 
8 Young T, Finn L, Peppard PE, et al. Sleep disordered breathing and mortality: eighteen-year follow-up of the Wisconsin sleep cohort. Sleep 2008; 31: 1071-1078.

9 Levy P, Pepin JL, McNicholas WT. Should all sleep apnoea patients be treated? Yes. Sleep Med Rev 2002; 6: 17-26.

10 Lavie P, Lavie L. Unexpected survival advantage in elderly people with moderate sleep apnoea. J Sleep Res 2009; 18: 397-403.

11 Yaffe K, Laffan AM, Harrison SL, et al. Sleep-disordered breathing, hypoxia, and risk of mild cognitive impairment and dementia in older women. JAMA 2011; 306: 613-619.

12 Johns MW. A new method for measuring daytime sleepiness: the Epworth sleepiness scale. Sleep 1991; 14: 540-545.

13 Carskadon MA, Dement WC, Mitler MM, et al. Guidelines for the multiple sleep latency test (MSLT): a standard measure of sleepiness. Sleep 1986; 9: 519-524.

14 Zung WW. A self-rating depression scale. Arch Gen Psychiatry 1965; 12: 63-70.

15 Spielberger CD, Gorsuch RL, Lushene R, et al. eds. Manual for the State-Trait Anxiety Inventory (Form Y). Palo Alto, Consulting Psychologists Press Inc., 1983.

16 Palta M. Quantitative Methods in Population Health: Extensions of Ordinary Regression. New Jersey, John Wiley \& Sons, Inc, 2003.

17 Sforza E, Roche F, Thomas-Anterion C, et al. Cognitive function and sleep related breathing disorders in a healthy elderly population: the SYNAPSE study. Sleep 2010; 33: 515-521.

18 Martinez-Garcia MA, Campos-Rodriguez F, Catalan-Serra P, et al. All cause and cardiovascular mortaility in elderly patients with sleep apnea. Role of CPAP treatment. A 6-year follow-up study. Am J Respir Crit Care Med 2011; 183: A1058.

19 Gooneratne NS, Richards KC, Joffe M, et al. Sleep disordered breathing with excessive daytime sleepiness is a risk factor for mortality in older adults. Sleep 2011; 34: 435-442.

20 Ancoli-Israel S, Kripke DF, Klauber MR, et al. Morbidity, mortality and sleep-disordered breathing in community dwelling elderly. Sleep 1996; 19: 277-282.

21 Phillips BA, Berry DT, Lipke-Molby TC. Sleep-disordered breathing in healthy, aged persons. Fifth and final year follow-up. Chest 1996; 110: 654-658.

22 Empana JP, Dauvilliers Y, Dartigues JF, et al. Excessive daytime sleepiness is an independent risk indicator for cardiovascular mortality in community-dwelling elderly: the three city study. Stroke 2009; 40: 1219-1224.

23 Morrell MJ, Jackson ML, Twigg GL, et al. Changes in brain morphology in patients with obstructive sleep apnoea. Thorax 2010; 65: 908-914.

24 Ayalon L, Ancoli-Israel S, Drummond SP. Obstructive sleep apnea and age: a double insult to brain function? Am J Respir Crit Care Med 2010; 182: 413-419.

25 Gozal D, Row BW, Kheirandish L, et al. Increased susceptibility to intermittent hypoxia in aging rats: changes in proteasomal activity., neuronal apoptosis and spatial function. J Neurochem 2003; 86: 1545-1552.

26 Decary A, Rouleau I, Montplaisir J. Cognitive deficits associated with sleep apnea syndrome: a proposed neuropsychological test battery. Sleep 2000; 23: 369-381.
27 Baldwin CM, Kapur VK, Holberg CJ, et al. Associations between gender and measures of daytime somnolence in the Sleep Heart Health Study. Sleep 2004; 27: 305-311.

28 Valipour A, Lothaller H, Rauscher $\mathrm{H}$, et al. Gender-related differences in symptoms of patients with suspected breathing disorders in sleep: a clinical population study using the sleep disorders questionnaire. Sleep 2007; 30: 312-319.

29 Young T, Hutton R, Finn L, et al. The gender bias in sleep apnea diagnosis. Are women missed because they have different symptoms? Arch Intern Med 1996; 156: 2445-2451.

30 Sforza E, Chouchou F, Collet P, et al. Sex differences in obstructive sleep apnoea in an elderly French population. Eur Respir J 2011; 37: 1137-1143.

31 Malhotra A, Huang Y, Fogel R, et al. Aging influences on pharyngeal anatomy and physiology: the predisposition to pharyngeal collapse. Am J Med 2006; 119: 72 e9-e14.

32 Kollias I, Krogstad O. Adult craniocervical and pharyngeal changes - a longitudinal cephalometric study between 22 and 42 years of age. Part I: Morphological craniocervical and hyoid bone changes. Eur J Orthod 1999; 21: 333-344.

33 Erskine RJ, Murphy PJ, Langton JA, et al. Effect of age on the sensitivity of upper airway reflexes. Br J Anaesth 1993; 70: 574-575.

34 Eikermann M, Jordan AS, Chamberlin NL, et al. The influence of aging on pharyngeal collapsibility during sleep. Chest 2007; 131: 1702-1709.

35 Mathur R, Douglas NJ. Frequency of EEG arousals from nocturnal sleep in normal subjects. Sleep 1995; 18: 330-333.

36 Kobayashi M, Namba K, Tsuiki S, et al. Clinical characteristics in two subgroups of obstructive sleep apnea syndrome in the elderly: comparison between cases with elderly and middle-age onset Chest 2010; 137: 1310-1315.

37 Kostikas K, Browne HA, Ghiassi R, et al. The determinants of therapeutic levels of continuous positive airway pressure in elderly sleep apnea patients. Respir Med 2006; 100: 1216-1225.

38 Vazir A, Hastings PC, Dayer M, et al. A high prevalence of sleep disordered breathing in men with mild symptomatic chronic heart failure due to left ventricular systolic dysfunction. Eur J Heart Fail 2007; 9: 243-250.

39 Bassetti C, Aldrich MS, Chervin RD, et al. Sleep apnea in patients with transient ischemic attack and stroke: a prospective study of 59 patients. Neurology 1996; 47: 1167-1173.

40 Arzt M, Young T, Peppard PE, et al. Dissociation of obstructive sleep apnea from hypersomnolence and obesity in patients with stroke. Stroke 2010; 41: e129-e134.

41 Hastings PC, Vazir A, O'Driscoll DM, et al. Symptom burden of sleep-disordered breathing in mild-to-moderate congestive heart failure patients. Eur Respir J 2006; 27: 748-755.

42 Johns MW. Sensitivity and specificity of the multiple sleep latency test (MSLT), the maintenance of wakefulness test and the epworth sleepiness scale: failure of the MSLT as a gold standard. J Sleep Res 2000; 9: 5-11.

43 Mediano O, Barcelo A, de la Pena M, et al. Daytime sleepiness and polysomnographic variables in sleep apnoea patients. Eur Respir J 2007; 30: 110-113. 